\title{
Concurrence des marchés de main-d'œuvre et dumping social dans l'agriculture
}

\author{
Béatrice Mesini et Catherine Laurent
}

\section{(2) OpenEdition}

\section{Journals}

\section{Édition électronique}

URL : http://journals.openedition.org/economierurale/4766

DOI : $10.4000 /$ economierurale.4766

ISSN : 2105-2581

\section{Éditeur}

Société Française d'Économie Rurale (SFER)

\section{Édition imprimée}

Date de publication : 15 décembre 2015

Pagination : 171-176

ISSN : 0013-0559

\section{Référence électronique}

Béatrice Mesini et Catherine Laurent, « Concurrence des marchés de main-d'œuvre et dumping social dans l'agriculture », Économie rurale [En ligne], 349-350 | septembre-novembre 2015, mis en ligne le 15 décembre 2017, consulté le 20 avril 2019. URL : http://journals.openedition.org/economierurale/4766 ; DOI : 10.4000/economierurale.4766 


\section{Concurrence des marchés de main-d'œuvre et dumping social dans l'agriculture}

La rubrique « Débat » d'Économie rurale s'appuie sur des interventions dans le cadre des Séminaires de politiques agricoles organisés par la Société française d'économie rurale. Sur un sujet d'actualité, deux points de vue courts sont demandés aux intéressés. Cette fois-ci, il s'agit de développer la question des marchés de main-d'œuvre et du développement du dumping social dans un contexte de mise en concurrence des exploitations agricoles. Deux auteures, Béatrice Mésini et Catherine Laurent, se sont prêtées au jeu, sur la base de leur intervention du 18 novembre 2014.

\section{Le détachement transnational dans} l'agriculture provençale : distorsion concurrentielle des marchés productifs et optimisation sociale des marchés du travail en Europe

Point de vue de Béatrice Mesini

Géographe, CNRS-Telemme et Aix-

Marseille Université

Peu étudiée en raison de son inscription récente et éphémère dans l'espace rural, la main-d'œuvre latino-américaine détachée dans le département des Bouches-du-Rhône, s'affirme à la fois comme puissant facteur de productivité des exploitations françaises utilisatrices, comme variable d'ajustement de la compétitivité agricole des pays européens et comme nouveau système de placement de la main-d'œuvre étrangère par les entreprises, en lieu et place des États. Depuis les années 2000, des entreprises de travail temporaire espagnoles, spécialisées dans le travail en agriculture, mettent à disposition des travailleurs temporaires extracommunautaires dans l'agriculture européenne. L'entreprise de travail temporaire espagnole Terra Fecundis, qui détachait 778 travailleurs andins dans le département des Bouches-du-Rhône, 2004, en met 2318 à disposition de 180 exploitations arboricoles et maraîchères en 2012.

En Espagne, le service juridique de l'ETT prend en charge et gère l'ensemble des droits civils et politiques des salariés détachés, principalement équatoriens, mais aussi boliviens, colombiens et péruviens : les demandes de permis de travail et de résidence, les visas, les déclarations de revenu, la nationalité espagnole, le regroupement familial, les cartes d'étudiants, le rapatriement... Simplicité, flexibilité et productivité sont les clés du succès de cette nouvelle forme d'emploi. Facturée entre 13 et 15 euros de l'heure à l'entreprise utilisatrice ${ }^{1}$, la main-d'œuvre détachée est plus onéreuse que celle introduite contractuellement par l'Office Français de l'Immigration et de l'Intégration', mais elle est jugée plus rentable puisque son volume est strictement ajusté aux besoins et aux aléas de la production et qu'elle assure l'augmentation du rendement productif des équipes. Pour les entreprises utilisatrices, l'externalisation fait partie d'une nouvelle stratégie d'organisation qui consiste à transférer tout ou partie de la gestion des ressources humaines à un partenaire extérieur.

1. Contre 20 à 21 euros pour une entreprise d'intérim française, qui bénéficie pourtant d'une exonération totale d'impôt sur les travaux saisonniers.

2. La durée totale du (ou des) contrats saisonniers dont un travailleur étranger est susceptible de bénéficier ne peut excéder six mois par an (article R 5221-23 du Code du travail). 
Les grandes exploitations du département emploient davantage de maind'œuvre à l'hectare, car on y trouve $90 \%$ du verger de pêchers de PACA et $70 \%$ de celui d'abricotiers ${ }^{3}$. L'étude menée sur les comptes 2010 de 24 exploitations fruitières et maraîchères du département par le député $B$. Reynès, montre que les « coûts de main-d'œuvre des arboriculteurs représentent $40 \%$ de leurs charges totales et que pour plusieurs entreprises ce rapport dépasse $50 \% »^{4}$. Le rapport dénonce les « distorsions de concurrence », qui ne proviennent pas seulement des pays extraeuropéens à bas coût de main-d'œuvre, mais aussi des États membres où le coût du travail est inférieur, tels que l'Espagne ou l'Allemagne. Autre composante de la concurrence déloyale, celle des pays extraeuropéens comme le Chili ou l'Argentine où le salaire minimum est inférieur à $300 €$, dont les fruits arrivent sur le marché français à des prix inférieurs à ceux des producteurs locaux, malgré les coûts de transport ${ }^{5}$.

En Espagne, la première disposition de l'article 17 de la loi 14/1994 qui porte sur les déplacements temporaires de travailleurs dans un État membre de l'Union européenne effectués par des entreprises établies dans le pays, indique qu'elles « devront leur garantir les conditions de travail répondant aux normes des pays de mise à disposition, sans préjudice de l'application de conditions de travail plus favorables découlant des dispositions de leur contrat de travail, des conventions collectives ou des contrats individuels ». Plus récente, la loi de réforme du marché du travail conduite en 2012, consacre la réussite des entreprises de travail temporaire, qui ont suppléé à la

3. Source Agreste PACA (2008). Enquêtes Vergers. Étude $\mathrm{n}^{\circ} 39$, novembre, p. 2.

4. Bernard Reynès (2011). Étude et propositions concernant les enjeux du coût de main-d'œuvre dans le secteur de la production agricole. Rapport au Premier ministre, Assemblée nationale, juin, pp. 15-16.

5. Op. cit. Rapport Reynès, pp. 16 et 18. carence de l'État: «Les services publics de l'emploi se sont montrés insuffisants dans la gestion du placement, contrairement aux entreprises de travail temporaire qui se sont révélées comme puissants agents dynamisateurs du marché du travail. ${ }^{6}$

En France, le statut des salariés détachés a été modifié par une loi du 17 décembre 2012, qui prévoit que les employeurs détachant temporairement des salariés sur le territoire national sont soumis aux dispositions légales et conventionnelles applicables aux salariés employés par les entreprises de la même branche d'activité 7 . Une entreprise qui recourt à une prestation de service internationale doit vérifier au moment de la conclusion du contrat commercial que le prestataire est bien immatriculé, qu'il a effectué la déclaration nécessaire auprès des services de l'inspection du travail, que les salariés détachés sont régulièrement déclarés dans leur pays d'origine, qu'ils bénéficient de la protection sociale et d'une rémunération au moins égale à celle qu'ils percevraient en France.

Toutefois, en matière de sécurité sociale, la compétence européenne ne s'exerce que pour opérer une coordination entre les systèmes de différents États membres, de manière à assurer la continuité des droits des bénéficiaires. Le choix a été fait du maintien de l'affiliation dans le pays d'origine, pour éviter de fréquentes affiliations et désaffiliations. Or, selon les témoignages collectés dans la Crau depuis 2006, ces droits ne sont guère respectés : il n'y a pas de suivi médical en Espagne, avant ou au terme des contrats, pas plus qu'en France où chacun se débrouille pour se soigner. Par ailleurs, les travailleurs ne reçoivent aucune protection de travail durant leurs missions. En outre, l'entreprise se rémunère sur les

6. Ley 3/2012, de 6 de julio 2012, de medidas urgentes para la reforma del mercado laboral, consultée le 20 février 2013 sur http://www.boe. es/diario_boe/txt.php?id=BOE-A-2012-9110.

7. $C f$. article L1262-4 du Code du travail. 
services fournis aux salariés : 200 euros l'aller-retour en bus France-Espagne, 35 euros pour transférer des mandats aux familles en Équateur, alors que la loi espagnole 3/2012 précise que « lorsque les ETT agissent en tant qu'agences de placement, elles doivent se conformer à l'obligation d'assurer aux travailleurs la gratuité pour la prestation de services ».

Enfin, les travailleurs interviewés sont payés 7,40 euros de l'heure, sans distinction de jours fériés ou de week-ends, alors que la législation française prévoit que les salariés détachés doivent être rémunérés sur la base du SMIC (brut horaire fixé à 9,53 euros en 2014) ou du salaire minimum conventionnel s'il est plus élevé. Même si la totalité des heures effectuées en France leur est versée, les travailleurs détachés s'interrogent sur les effets d'une sousdéclaration puisque seuls 10 à 13 jours figurent sur leur fiche de paie en Espagne, alors qu'ils travaillent en moyenne entre 22 à 24 jours par mois. En France, pourrait être caractérisé le délit de travail dissimulé par « dissimulation de tout ou partie d'un emploi salarié » qui vise expressément «la mention d'un nombre d'heures inférieur à celui réellement effectué sur le bulletin de paie », délit puni de trois ans de prison et de 45000 euros d'amende. Se présume aussi une sous-déclaration de cotisations des entreprises utilisatrices, sachant que la déclaration sur la valeur ajoutée doit indiquer le nombre de salariés employés, et que celles qui y sont assujetties doivent déclarer les salariés détachés par un employeur établi hors de France.

Le Rapport d'information relatif à l'exécution de la directive européenne sur le détachement des travailleurs 2013, questionne les vertus du modèle de libre circulation des biens et des personnes, qui induit des «effets socio-économiques pervers » en contexte de crise : «En particulier, on observe des dérives, des changements d'objet et des détournements de la directive, au profit de politiques systématiques et délibérées d'optimisation sociale, visant à obtenir un avantage concurrentiel au sein du marché intérieur européen, qui ont des conséquences particulièrement préoccupantes. $»^{8}$

Anticipant la transposition de la directive qui encadre le détachement au sein de l'UE', la loi française du 10 juillet 2014 a entrepris de " lutter contre la concurrence sociale déloyale », en renforçant les contrôles et sanctions contre les entreprises qui ont recours de "manière abusive » à des travailleurs détachés. Le texte instaure le principe de responsabilité solidaire, permettant de poursuivre l'entreprise donneuse d'ordre en cas de fraude et fait peser sur elle une obligation de vigilance en matière de respect du droit du travail et de rémunération des travailleurs détachés ${ }^{10}$, puisqu'elle sera tenue avec l'employeur au paiement des rémunérations, indemnités et charges en cas d'irrégularité. Enfin, la loi crée une liste noire des entreprises condamnées pour travail illégal, qui pourront se voir refuser le bénéfice de toute aide publique pour une durée de 5 ans.

\section{Salariat agricole et dumping social : goodbye Chayanov ?}

\section{Point de vue de Catherine Laurent}

Économiste, INRA-SAD

Selon les derniers Recensements de l'agriculture (RA), qui constituent la source la

8. Savary G., Guittet C., Piron M. (2013). Rapport d'information déposé par la Commission des Affaires européennes, relatif à l'exécution de la directive sur le détachement des travailleurs, enregistré à la Présidence de l'Assemblée nationale, le 29 mai, pp. 6-7.

9. Adoptée par le Parlement européen le 16 avril 2014.

10. En cas de non-paiement du salaire minimum à un salarié ou de non-respect du temps de repos obligatoire, des amendes pourront être infligées, pouvant aller jusqu'à 2000 euros par travailleur détachés, et jusqu'à 4000 euros en cas de récidive. 
plus complète de données sur les actifs agricoles (EC, 2013) $)^{11}$, l'UE comptait 12 millions d'exploitations agricoles en 2010 et 25 millions de personnes ayant une activité agricole « régulière » dont $92,2 \%$ de main-d'œuvre familiale et 7,8 \% de salariés " permanents » (en contrat à durée indéterminée). Un nombre indéfini de salariés agricoles «non permanents » s'ajoute à ces effectifs. En France, la proportion relative de travail fourni par les salariés dans les exploitations entre 1979 et 2010 (RA) s'est accrue : de 11 à $17 \%$ du total des Unités de travail annuel (UTA) recensées pour les salariés « permanents », de 5 à $12 \%$ pour les salariés «non permanents » et les personnes intervenant sous forme de prestation de service. Mais il est impossible de savoir à combien de personnes physiques correspond une UTA déclarée, car pour cette catégorie de travailleurs, la statistique agricole dissocie le travail du travailleur et ne fournit que le décompte agrégé, sous forme d'UTA, du travail déclaré par les exploitants pour ces personnes.

Un recoupement simple de données administratives (déclarations de contrats à durées déterminées) (voir par exemple pour la France : S. Villaume, 2011) ${ }^{12}$ montre que cela concerne à coup sûr plusieurs millions de personnes en Europe. Mais combien exactement? Cela reste indéterminé. Les difficultés viennent de ce qu'il ne semble pas exister au ministère de l'Agriculture ou à la Mutualité sociale agricole de base de données nationale de l'ensemble des personnes ayant des contrats à durée déterminée en France, sans double compte entre régions. Or un salarié «non permanent »

11. EC (European Commission) 2013. How many people work in agriculture in the European Union? An answer based on Eurostat data sources. $E U$ agriculture economic briefs, $\mathrm{n}^{\circ} 8,17 \mathrm{p}$.

12. Villaume S. (2011). L'emploi salarié dans le secteur agricole : le poids croissant des contrats saisonniers, Insee première, $\mathrm{n}^{\circ} 1368$. peut être embauché quelques jours seulement, ou pendant plusieurs mois d'affilée via une succession de " contrats saisonniers » sur une même exploitation ou en circulant entre plusieurs zones de production.

De plus, le nombre d'UTA fournies par les salariés « non permanents » est sous-estimé du fait de sous-déclarations importantes. Ceci concerne aussi bien les personnes directement payées par les exploitations que celles payées par des tiers. Ces constats sont partagés par les diverses institutions en charge du suivi de l'emploi des salariés agricoles (par exemple Commission contre le travail illégal 2013 ; DGT, 2012) $)^{13}$.

Ces difficultés de décompte sont accentuées par le fait que les personnes ne bénéficiant pas d'un emploi permanent sont embauchées sous des statuts extrêmement divers par les exploitants (en France contrats à durée déterminée classiques mais aussi saisonniers, contrats vendange, stagiaires, apprentis, woofing...) ou par des tiers fournissant de la main-d'œuvre aux exploitations sous forme de prestation de service (agences d'intérim, entreprises de travaux agricoles, prestataires employant des travailleurs détachés, etc.).

Cette situation n'est pas entièrement nouvelle ni spécifique à la France. Cependant, un ensemble convergent d'informations - obtenues par des travaux de recherche, conduits principalement par des sociologues (Mesini 2008 ; Morice, Michalon 2008 ; Décosse, 2011 ; Nguyen,

13. Commission Nationale de Lutte contre le Travail Illégal (2013). Bilan du Plan National de Lutte contre le Travail Illégal 2013-2015 Perspectives 2014, 5 décembre, 38 p. ; DGT (Direction générale du travail) (2012). Analyse des déclarations de détachement des entreprises prestataires de service en France en 2011, $65 \mathrm{p}$. 
Purseigle 2012... $)^{14}$, et par les services déconcentrés du ministère en charge du travail - montre une profonde transformation des statuts d'emploi des salariés agricoles, qui s'accompagne dans de nombreux cas d'une précarisation accrue des personnes concernées (Laurent, 2013) ${ }^{15}$.

$\mathrm{Si}$ cette précarisation est constatée, force est d'observer que l'intervention publique pour la combattre reste, pour le moins, modérée. Certaines réglementations qui ont tenté d'y remédier échouent à améliorer la situation sans susciter de réajustement. Ainsi, dans une étude sur les groupements d'employeurs, Elyakime $(2007)^{16}$ a montré que ces dispositifs ont surtout été un support de précarisation par les types de contrats qu'ils proposent (plus de $70 \%$ de CDD). D'autres réglementations encouragent explicitement la précarité ainsi que le montre le texte précédent de Béatrice Mesini qui revient plus en détail sur la question des travailleurs détachés, ou ainsi qu'en atteste la loi du 11 juillet 2014 qui accorde un statut dérogatoire aux employeurs du monde agricole

14. Mesini B. (2008). Flexi-insécurité dans un secteur en tension : processus de segmentation statutaire et éthique du marché des saisonniers étrangers dans l'agriculture. Revue Asylon, $\mathrm{n}^{\circ} 4$, 20 p. ; http://www.resear-terra.eu/article.html ; Morice A., Michalon B. (2008). Introduction numéro spécial « Les migrants dans l'agriculture: vers une crise de main-d'œuvre? » Études rurales, $\mathrm{n}^{\circ}$ 182, pp. 9-28 ; Décosse F. (2011). Migrations sous contrôle : agriculture intensive et saisonniers marocains sous contrat "OMI ». Thèse de doctorat en sociologie. EHESS ; Nguyen G., Purseigle F. (2012). Les exploitations agricoles à l'épreuve de la firme. L'exemple de la Camargue. Études rurales, ${ }^{\circ} 190$, pp. 99-118.

15. Laurent C. (2013). French Mediterranean Agriculture: Images of the multifunctional agriculture to mask social dumping? Research in Rural Sociology and Development, Emerald group Publishing Limited, vol. 19, pp. 149-171.

16. Elyakime B. (2007). Groupements d'employeurs agricoles : quelle aide publique locale ? Revue d'Économie Régionale et Urbaine, pp. 861-880. à l'obligation d'indemniser des stages supérieurs à deux mois... La liste n'est pas exhaustive.

Ces constats sont loin d'être anecdotiques, car ces évolutions (i) concernent un nombre croissant de personnes, (ii) s'accompagnent d'une dégradation des conditions de rémunération qui concernent aussi bien les salaires directs qu'indirects (protection sociale, santé et sécurité au travail...), (iii) et deviennent structurelles dans la constitution de la compétitivité du secteur, notamment dans les sous-secteurs à forte composante de main-d'œuvre (arboriculture, maraîchage...).

Ces constats invitent ainsi à développer un agenda de recherche sur le travail agricole qui permettrait de documenter, de façon articulée, plusieurs dimensions.

Il s'agit d'abord de mieux relier l'évolution des formes de concurrence internationale et les nouvelles formes d'exploitation du travail salarié agricole dans l'analyse du fonctionnement du secteur. À titre d'exemple, un rapport récent de l'Organisation internationale du travail estime à 6,4 milliards de dollars les profits annuels supplémentaires réalisés par les employeurs agricoles des pays développés, du fait du seul recours au « travail forcé », limite extrême de l'exploitation liée à la précarisation des personnes (ILO, 2014) $)^{17}$.

Il s'agit ensuite de réfléchir à la soutenabilité politique, pour la France et pour l'Europe, d'une mise en concurrence entre travailleurs dans des conditions où le recrutement dans le respect du code du travail de salariés résidant en France est plus coûteux que l'achat de prestations de service assurées par des salariés détachés dont la protection sociale est payée dans le pays d'origine (différentiel estimé à $30 \%$ en

17. ILO (International Labour organisation) (2014). Profit and poverty. The economics of forced labour. $57 \mathrm{p}$. 
moyenne par Bocquet $(2013)^{18}$, et analyser les limites à terme de solutions de substitution trouvées jusque-là (employeurs exonérés du paiement de leur part de cotisations sociales pour les salariés occasionnels).

Il s'agit enfin de réfléchir aux conséquences structurelles sur l'agriculture familiale de ces nouvelles formes d'exploitation du travail. Et la réflexion ne peut s'arrêter au salariat. C'est l'ensemble du modèle de l'exploitation familiale qui doit être considéré.

En effet, les thèses d'Alexandre Chayanov $^{19}$, économiste russe du début du $\mathrm{XX}^{\mathrm{e}}$ siècle, furent souvent mobilisées pour expliquer la persistance d'une petite agriculture familiale alors même que d'autres secteurs d'activité basculaient dans un salariat généralisé. Et, de fait, de nombreuses observations ont confirmé que des paysans travaillant pour leur propre compte pouvaient passer le cap d'années difficiles, en réduisant leur consommation en deçà de ce que permettrait un salaire minimum mensuel, en allongeant leurs journées de travail et celles de leur entourage familial au-delà des normes salariales du moment, lorsque la nécessité s'en fait ressentir. Autant d'ajustements débouchant sur une auto-exploitation à certains égards plus radicale que celle permise par les rapports de subordination du salariat.

En France, depuis les lois d'orientation agricole du début des années 1960, les interventions publiques ont visé à la fois à garantir une certaine parité de revenu entre exercice d'une activité agricole indépendante à plein temps et exercice d'une activité salariée. Ceci concernait les revenus directs (revenu net/salaire) mais aussi les rémunérations indirectes (protection sociale, système de santé et sécurité au travail, droits à la retraite...). Les normes de ces rémunérations indirectes sont désormais constitutives du métier d'agriculteur, en raison notamment de l'évolution des organisations du secteur (régime d'assurance et de protection sociale pour tous les actifs du secteur, normes de sécurité au travail...). Dans ce domaine des rémunérations indirectes en France, les ajustements d'autoexploitation du travail paysan conceptualisés par Chayanov jouent peu et ne peuvent être source de résilience, car les principaux coûts associés (cotisations sociales, primes d'assurance...) sont encadrés par des règles collectives et obligatoires.

Le recours à des emplois tels que les travailleurs détachés modifie donc les conditions de mise en concurrence de toutes les personnes travaillant dans l'agriculture en construisant la compétitivité du secteur sur le contournement d'institutions qui ne peuvent faire l'objet d'ajustements individuels ni par les salariés résidant en France ni par les travailleurs indépendants de petites exploitations familiales dont la survie dépend de la possibilité d'ajustements tels que décrits par Chayanov. La précarisation de l'emploi agricole apparaît ainsi comme un élément clé de l'évolution structurelle actuelle de l'agriculture.

18. Bocquet E. (2013). Rapport d'information fait au nom de la commission des affaires européennes sur les normes européennes en matière de détachement des travailleurs. Rapport $\mathrm{n}^{\circ}$ 527, Sénat, Session ordinaire de 2012-2013.

19. Chayanov A. (1990). L'organisation de l'économie paysanne. Paris, Librairie du Regard. 